\title{
EL DIPLOMA EN EDUCACIÓN EN MODALIDAD A DISTANCIA. EVALUACIÓN DE UNA EXPERIENCIA PILOTO
}

\author{
Dr. Alejandro Armellini*
}

\begin{abstract}
El Diploma en Educación es un postgrado de un año de duración, ofrecido por el Instituto de Educación de la Universidad ORT Uruguay desde el año 1996 y dirigido a docentes en actividad. Conjuntamente con su quinta edición en modalidad presencial, en abril de 2000 comenzó la primera edición del Diploma en Educación en modalidad a distancia. Desde entonces, el Instituto de Educación ha ofrecido el Diploma en ambas modalidades, todos los años. El título obtenido por los participantes es el mismo, independientemente de la modalidad de estudio que elijan.

Por ser el primer postgrado para educadores dictado por una universidad uruguaya a través de Internet, se entendió necesario realizar un seguimiento de su desarrollo, en busca de conclusiones que pudieran aplicarse a éste y otros cursos en modalidad a distancia. El trabajo de seguimiento, que abarcó aspectos académicos, tecnológicos y administrativos, estuvo a cargo de Mirian Gregori y Alejandro Armellini, bajo la coordinación general de Enrique Lorenzo.

Este artículo presenta una síntesis de los resultados de carácter académico que se desprenden del estudio de seguimiento del Diploma en Educación (modalidad a distancia, edición 2000-2001) y las recomendaciones que surgen de dicho estudio.
\end{abstract}

\section{El Diploma en Educación}

Para cursar el Diploma en Educación en la apuntada modalidad, los candidatos deben reunir requisitos de dos tipos: académicos (poseer título docente o universitario y experiencia docente relevante) y tecnológicos. Los segundos incluyen el manejo fluido de herramientas básicas de informática (procesadores de texto, correo electrónico, navegación por Internet) y la disponibilidad de acceso regular y confiable a un computador personal conectado a Internet.

El alumno que se inscribe en este postgrado asiste a una sesión inicial presencial en el Instituto de Educación, en la que recibe un conjunto de libros "de cabecera", el cronograma del curso y documentación diversa relativa a aspectos administrativos y académicos del postgrado. En este primer contacto se brinda una introducción a las herramientas informáticas que se utilizarán durante el curso, particularmente aquellas a las que el participante no está acostumbrado. La Universidad ORT utiliza un software de gerenciamiento de cursos a distancia llamado WebCT, cuyas funcionalidades principales son presentadas en esta instancia. A partir de esta visita inicial, el postgrado se desarrolla con Internet como plataforma principal de entrega. Además de las comunicaciones mediatizadas por esa vía, se utiliza el teléfono, el fax y el correo postal.

En muchas ocasiones, los participantes optan por visitar a docentes o tutores en el Instituto de Educación. Los participantes del Diploma en su modalidad a distancia no son exclusivamente residentes del interior del país. Varios de ellos viven en Montevideo y optan por cursar a distancia en virtud del manejo individual del tiempo que esta modalidad ofrece. 
El sitio web del curso contiene una guía de aprendizaje por seminario (que incluye lecturas y tareas), material de apoyo, links a otros sitios relevantes y bibliografía adicional. La propuesta se centra en la interactividad de los participantes con los contenidos y la interacción participantes-docentes y entre participantes. Esto ocurre fundamentalmente a través de la elaboración de aportes y reflexiones, ya sea trabajando individualmente o en equipo, sobre los temas presentados por las guías de aprendizaje y a partir de las lecturas, referencias y tareas incluidas en ellas. Estos aportes son "colgados" por los participantes en el foro de discusión de cada seminario. Es así que el foro de discusión actúa como articulador y eje de la propuesta.

Los seminarios, en este marco, no pretenden ser "cursos de autoestudio" ni "enlatados". Esto significa que no es suficiente para el participante leer la guía de aprendizaje y realizar las tareas allí contenidas. Por el contrario, resulta imprescindible ampliar los conceptos contenidos en la guía y agregar otros a partir de material adicional, reflexionando y compartiendo aportes a través, principalmente, del foro de discusión.

Cada seminario es evaluado individualmente. La aprobación de todos los seminarios constituye un requisito para realizar el proyecto, que culmina con la elaboración de un informe de investigación o innovación educativa y una defensa presencial.

\section{El contexto estudiado}

La edición del postgrado que fue objeto de nuestro estudio tuvo una estructura de cinco seminarios a distancia de carácter obligatorio (abril a agosto de 2000), dos seminarios optativos (octubre y noviembre de 2000) y un trabajo tutoreado de investigación o innovación educativa (agosto de 2000 a marzo de 2001). Este trabajo culminó con la redacción de un informe de investigación o innovación y su defensa presencial.

El siguiente cuadro muestra los seminarios que formaron parte del postgrado analizado, cuya versión presencial insume 180 horas de clase:

\begin{tabular}{|l|l|}
\hline Seminarios obligatorios & Seminarios optativos \\
\hline - Innovación y cambio educativo & \\
- Concepciones de la enseñanza & \\
- Concepciones del aprendizaje & - Cómo escribir un artículo técnico \\
- El proceso de investigación/innovación I $\left.*^{*}\right)$ & - El proceso de Investigación/innovación II $\left(^{*}\right)$ \\
- Metodologías de investigación & \\
\hline
\end{tabular}

(*) Dictados por un profesor invitado de la Universidad de Harvard en modalidad presencial. Asistieron tanto los alumnos presenciales como los alumnos a distancia.

Una vez concluidos los seminarios, los participantes se dividieron en grupos de 2 ó más personas, en función de intereses y objetivos, a efectos de iniciar el trabajo de investigación o innovación. La temática elegida por cada equipo determinó el tutor asignado, como es habitual en este postgrado. La tutoría consistió en reuniones periódicas entre los alumnos y el tutor. Estos encuentros ocurrieron en modalidad presencial, telefónicamente o fueron mediatizados por Internet.

El número de alumnos en esta edición del Diploma fue 34. Se registraron tres deserciones. Cinco fueron los docentes a cargo del dictado de los seminarios, cuatro de los cuales realizaron, además, tutorías. Hubo un quinto tutor que no tuvo a su cargo ninguno de los seminarios. 
El estudio de seguimiento analizó los dos grandes componentes del curso: seminarios y tutorías. Este artículo presenta los aspectos académicos más salientes del análisis de los seminarios y realiza las recomendaciones que de él surgen.

\section{El estudio de seguimiento y evaluación del Diploma en Educación en modalidad a distancia (2000-2001)}

\section{Objetivos generales del estudio}

a. Realizar una evaluación sistemática del dictado del Diploma en Educación en su modalidad a distancia (edición 2000-2001).

b. Extraer la información relevante de dicha evaluación para ser transferida y aplicada en futuras ediciones de este postgrado y de otros cursos a dictarse en esta modalidad en la Universidad ORT Uruguay.

\section{Diseño metodológico}

Se trató de un estudio de caso de corte mixto, cuantitativo y cualitativo. Se buscó causar la menor alteración posible a la labor de los involucrados y a la dinámica del Instituto de Educación en su conjunto, sin menoscabar la calidad de información recogida con relación a los objetivos del estudio.

Los datos para el análisis provienen de:

1. Cuestionarios electrónicos entregados por los alumnos del programa (*).

2. Cinco entrevistas pautadas a los docentes a cargo de los seminarios.

3. Cuatro cuestionarios electrónicos entregados por los tutores.

4. Una entrevista pautada a cada uno de los dos informantes claves.

5. Consultas al servidor web del curso.

(*) A efectos de analizar separadamente los seminarios de las tutorías, dos cuestionarios diferentes fueron administrados en diferentes momentos. Se recogieron 22 cuestionarios referidos a los seminarios que componen el postgrado y 21 cuestionarios sobre las tutorías. En este artículo se presentan únicamente los resultados referidos a los seminarios.

\section{Resultados}

\section{a. Requisitos de ingreso}

Si bien es relativamente sencillo establecer si un potencial participante reúne los antecedentes académicos, existen otros requisitos, críticos para quien participa de una propuesta a distancia como ésta, cuyo cumplimiento resulta de difícil verificación en un contexto geográficamente disperso. Un ejemplo de ello son los aspectos relativos al acceso a Internet y al manejo de la tecnología. En la edición estudiada, varios candidatos, en su afán de participar de este postgrado, declararon inicialmente reunir todos los requisitos de ingreso establecidos. Poco después de comenzar, resultó claro que esto no era así. Los datos siguientes, tomados de cuestionarios a alumnos (codificados como $C A$ ) ilustran esta situación:

"En verdad no puedo [evaluar la herramienta], porque no la usé". (CA 2)

"Perdida al inicio, por desconocimiento [del] funcionamiento [de la herramienta informática] y no hábito de uso". (CA 14) 
Los datos reunidos a partir de las consultas al servidor demuestran que hubo dos participantes que nunca accedieron al curso (CA 2 es uno de ellos). Dos estudiantes accedieron por primera vez en agosto y setiembre respectivamente, es decir, cuando ya habían finalizado todos los seminarios. Finalmente, encontramos un grupo de cinco alumnos con un total de entre tres y once accesos cada uno. Esto denota la utilización de medios alternativos para el acceso al material y para establecer comunicaciones con el resto del grupo y los docentes.

\section{b. Lo académico}

El aspecto académico fue destacado como el punto más fuerte del Diploma por el $50 \%$ de los alumnos que completaron el cuestionario. Del 50\% restante, el $64 \%$ se manifiesta neutro (por no poder jerarquizar, por no responder o por señalar a más de un aspecto como el más fuerte), y el restante $36 \%$ señala a lo administrativo y organizativo como el punto más fuerte de este postgrado.

\section{Guías de aprendizaje y materiales de apoyo}

Los alumnos expresaron conformidad con las guías de aprendizaje. Los datos sugieren que éstas cumplieron adecuadamente su función, en tanto se argumentó que el material de apoyo no siempre resultó suficiente. La percepción es de un alto nivel de satisfacción general. Los siguientes datos, tomados de los cuestionarios a alumnos, ilustran esta situación:

"Las guías fueron nuestro bastón cotidiano, la relectura que hacíamos en ellas siempre nos hacían descubrir algo nuevo. Bien pensadas. [...] Más material de apoyo. Sugerencias de búsqueda de material en el tema de investigación específicamente". (CA 3)

Los docentes, por su parte, destacan la importancia del proceso de elaboración de los materiales en línea. La confección de estos materiales, de acuerdo con su percepción, los obligó a "sistematizar" el curso. Anteriormente, el material necesario se encontraba disperso y en diversos formatos (papel, transparencias, archivos electrónicos). La revisión general necesaria para la elaboración de las guías impactó positivamente en sus prácticas docentes, incluyendo el ámbito presencial.

Los profesores realizaron un conjunto de sugerencias con respecto a las guías y al material de apoyo que generaron, con el fin de mejorar su calidad y efectividad en futuras ediciones de este postgrado. Estas sugerencias pueden agruparse de la siguiente manera (datos tomados de las entrevistas a los profesores, $E P$ ):

- Incrementar la interactividad, en especial alumno-PC (EP 1).

- Mejorar el diseño, la diagramación y la amigabilidad de las páginas que componen las guías y los materiales de apoyo (EP 1, EP 2).

- A través de links apropiados, agregar material relacionado al seminario, ya residente en otros servidores web en todo el mundo (EP 2).

- Incrementar el número de tareas. Las actividades en general deben ser más "profundas y trabajadas" (EP 4, EP 5).

- Dosificar cautelosamente el material que se va colocando en el sitio (EP 3).

Con respecto al número de tareas y a su aporte, a través de los cuestionarios, también los alumnos manifiestan un deseo de incrementarlas. Por ejemplo:

"Como ha sido muy bueno recibir "tareas" prácticas para realizar, quizá incluir algunas más, hasta ahora las planteadas han sido importantes para reflexionar sobre las prácticas" (CA 17). 


\section{Las comunicaciones}

Las funcionalidades que registraron mayor utilización fueron las asincrónicas (correo electrónico y foro de debate). Esto es habitual en cursos de esta naturaleza. Con respecto al correo electrónico, solamente estamos en condiciones de realizar un análisis en función del número de mensajes enviados y recibidos por cada docente, ya que el carácter privado y confidencial de los contenidos transmitidos por este medio no lo permite. A partir de estos datos, observamos que los dos primeros seminarios (Innovación y cambio educativo y Concepciones de la enseñanza) registraron un alto tráfico de mensajes, en contraste con los otros tres. Precisamente, estos dos primeros seminarios, estuvieron a cargo de docentes de «alta dedicación», a diferencia de los restantes.

El foro de discusión constituyó el eje del dictado de este postgrado a distancia, en términos de comunicación e interacción entre alumnos y docentes. Se trata de una funcionalidad que posibilita implementar un conjunto de mecanismos asociados a la transposición didáctica.

A través del foro, los docentes ensayaron estrategias que impactaron en varias dimensiones:

- La percepción de los alumnos sobre la calidad del curso. El foro opera como la versión asincrónica del aula, o el "aula virtual". Un buen foro implica la percepción de buena calidad en el curso.

- La asignación de tareas a grupos de alumnos conformados por personas geográficamente distantes incidió positivamente en la motivación y cohesión del grupo, a pesar de lo difícil que resulta el "monitoreo" por parte del docente.

- La invitación, el desafío y la provocación de la reflexión por parte de los docentes generó ricos y frecuentes aportes de los participantes. El asincronismo ofrece la brecha necesaria para lograr ese espacio adicional de reflexión que no se da en el "tiempo real" de una clase presencial o de un chat. Sin embargo, no todos los intercambios son igualmente ricos. En opinión de algunos profesores, muchos de sus alumnos "hablan mucho y dicen poco, hay cháchara" (EP 3).

- El docente siente una sensación de ansiedad, ocasionada por el hecho de estar al frente de "grupos silenciosos" sin posibilidades de saber qué está ocurriendo del lado del alumno. Es interesante que la soledad y el aislamiento, normalmente asociados al estudiante distante, también fueron experimentados, de diferentes modos, por los docentes a cargo de este postgrado.

El número de chats fue muy variable entre un seminario y otro. Las autoridades del Instituto le sugirieron a la docente del primer seminario no usar esta herramienta, pues tanto los alumnos como ella misma se encontraban desarrollando sus primeras armas en el manejo de WebCT. En cambio, el dictado del segundo seminario del postgrado (Concepciones de la enseñanza) incluyó cuatro chats. El tercer seminario tuvo un chat, el último del curso.

Cualitativamente, en el seminario sobre Concepciones de la enseñanza se hizo un uso muy interesante de esta funcionalidad. Esto recibió comentarios favorables de los participantes. A través de las excelentes intervenciones del docente se consiguió una buena participación y fluida interacción. Se logró un gran aprovechamiento de estas instancias y en definitiva, una buena propuesta de enseñanza. Estas apreciaciones están basadas en un análisis del registro textual de los cuatro chats realizados por el docente de referencia, quien al respecto, comentó en su entrevista:

"El chat es un mini-seminario sobre las temáticas de mayor interés (estaban planificados). Se discute en forma pautada. Son las "paradas" que no necesariamente estaban planificadas en la hoja de ruta: "aquí nos detenemos porque necesitamos profundizar". Herramienta más adecuada para ese tipo de trabajo... Hubo 1 por semana con 10-12-15 personas, una participación bastante alta"(EP 2).

El docente que realizó solamente un chat durante su seminario, manifestó en su entrevista que le "encantó", porque "se parece a una clase presencial", donde hubo un buen nivel de participación con varias conversaciones a la vez, aunque sin generarle estrés (EP 3). 
Desde la óptica de los alumnos, se recogen percepciones positivas sobre los chats, así como alguna sugerencia:

"[Sugiero] algunos chats más. Fueron novedosos, excelentes guías de orientación de aprendizaje y ricos espacios para compartir" (CA 17).

"[Los aportes de los docentes vía foros, chats y mails] fueron excelentes, muy buenos" (CA 22).

Parece sorprendente que dado el éxito constatado en los cuatro chats celebrados en el segundo seminario del postgrado, no se haya optado por su uso en el resto de los seminarios. Esto tiene que ver con una serie de aspectos, a saber:

\section{Lo operativo}

Los estudiantes a distancia expresaron preferencia por los domingos de noche para realizar chats: resulta imposible exigir al cuerpo académico del Instituto un compromiso de esta naturaleza.

\section{Lo tecnológico}

El uso del chat requiere el manejo fluido de una funcionalidad específica, que si bien está dentro de WebCT, no es una de las herramientas de uso cotidiano. Adicionalmente, el docente debe pensar, elaborar y digitar rápidamente durante el chat, a efectos de poder mantenerse "en sintonía", manteniendo varias “conversaciones" en simultáneo.

\section{La propuesta de enseñanza}

El docente que organiza y lidera un chat debe contar con un repertorio adecuado de técnicas específicas y sensibles a esta herramienta. Esto implica, por ejemplo, haber pautado previamente, a través del foro, los contenidos y dinámica del chat. Cada chat exige, además, una cuidadosa preparación de la sesión, con una buena cantidad de material relevante digitado previamente en un procesador de texto, para poder "copiar y pegar" ágilmente durante el encuentro en vivo. Es preciso tener la capacidad de conducir varias discusiones en paralelo, saber cuándo y cómo cortar la proliferación de temas, de qué modo realizar una puesta en común, en qué punto lanzar un nuevo tema o argumentación y sacar el máximo provecho de los temas emergentes.

En definitiva, los chats funcionaron como buenos socializadores y motivadores del grupo. El estar "todos allí y a la vez" permitió a docentes y alumnos establecer puentes entre el entorno presencial y esta nueva modalidad de trabajo: si bien no había proximidad física, la simultaneidad los acercó a un contexto que les es conocido.

\section{c. El docente a distancia}

De acuerdo a los profesores a cargo de los seminarios, el rol del docente en esta modalidad cambia significativamente. Analizando las entrevistas, encontramos percepciones interesantes acerca del rol propiamente dicho y de cómo cada docente se sintió en este nuevo contexto:

"Fuerte intervencionismo del docente, a diferencia del modo presencial, por el tema de los plazos los seminarios se sugiere sean estudiados en determinados plazos (sincronismo), por ej. mayo para Didáctica. Se percibe la necesidad de conectarse varias veces en el día, incluso fines de semana, para responder rápidamente y atender a los alumnos... Muy positivo, no se sabe qué está ocurriendo y es difícil de controlar" (EP 2).

"Papel 'directivo', participaciones muy frecuentes y acotadas, a diferencia de participaciones presenciales que son abiertas. La intervención tuvo que ser concisa, con límites establecidos para aprovechar los tiempos acotados" (EP 2). 
"Rol más protegido detrás de la pantalla, más cómodo, más impune: el medio es el mensaje. Uno es unas letras que aparecen escritas, ellos no ven una foto ni sienten una voz. Eso te da una armadura extra que no te muestra en ese estado de desnudez pedagógica que significa exponerte frente a un grupo" (EP 3).

Algunos docentes reflexionaron sobre las maneras en que ellos intentaron adaptar sus estrategias a la nueva modalidad y sobre los problemas que debieron enfrentar, por ejemplo:

"Veta histriónica desaparece. Uno debe tratar de buscar el relacionamiento mediante otro tipo de discurso. Limitaciones me frenaban al sentarme en la computadora" (EP 5).

A partir del análisis, los elementos más salientes en cuanto al rol del docente a distancia son:

- Un rol más directivo, pero a la vez más protegido, más "cómodo" y más "impune".

- Cambio en su discurso y en los medios en que ese discurso llega a los alumnos.

- Sensación de pérdida de control.

- Intervenciones docentes con tiempos diferentes de la que es necesaria en la clase presencial. Debe realizar un mayor número de conexiones, aunque sean por lapsos más breves.

Al igual que en el contexto presencial, en esta modalidad, la solvencia académica en sí misma no es suficiente. Tampoco basta con ser un "buen docente presencial" e intentar "traducir estrategias" al entorno virtual. Es muy importante hacer notar que, hacia fines de 1999, esta modalidad de trabajo era novedosa para todos los integrantes del equipo académico del Diploma. Uno de ellos se encontraba participando en un curso de este tipo como alumno, pero nunca lo había hecho como docente o tutor. En ese sentido, el equipo demostró una gran flexibilidad y avidez por aprender, ensayando y adaptando estrategias en un contexto nuevo para ellos.

Consultados acerca de las características del "docente a distancia ideal", los estudiantes se expresan de la siguiente manera:

"Nivel académico adecuado. Disponibilidad de tiempo para las consultas. Calidez humana. Sentido práctico para la resolución de conflictos que en ocasiones la distancia agrava" (CA 1).

"Paciencia. Intuición. Ser capaz de trasmitir seguridad al alumno. Saber "escuchar" lo que el alumno está tratando de decirle. Hacerle percibir al alumno que "él está ahí" aunque no lo vea. Tener una buena formación en el área que enseña. Ser capaz de transformarse más que en un profesor en un amigo del alumno, del cual será 'guía'" (CA 6).

Del análisis de los datos surgen las siguientes características de un buen docente a distancia:

- solvencia académica

- disponibilidad para consultas: rapidez en responder y ofrecer feedback

- capacidad para orientar y motivar

- calidez humana

- adecuada capacidad de gestión de una clase no presencial

- habilidad para lograr la reflexión, a pesar de la distancia

- capacidad para comprender, a la distancia, las percepciones de los alumnos

- manejo fluido de las herramientas de comunicación (foro, chat, correo electrónico)

- liderazgo 
Resulta claro que el compromiso del docente con la innovación educativa, con el desarrollo profesional y con la institución es clave para el éxito de un programa de esta naturaleza.

\section{d. El Diploma en Educación y el desarrollo profesional de los participantes}

Muchos de los participantes no habrían podido acceder a un postgrado como el Diploma en Educación si éste no hubiera sido ofrecido en modalidad a distancia. Hay coincidencia en la valoración positiva de los participantes sobre este curso:

"Muy positiva, porque permite a los docentes del interior actualizarse, y me parece muy buena en el sentido de que no hay que venir tan seguido" (CA 2).

"Me posibilitó realizar un curso de Postgrado que de otro modo sería imposible. Fue un verdadero desafío, una experiencia enriquecedora, valiosa, con amplia apertura académica. Espero poder realizar nuevos cursos en esta modalidad a distancia. ¡GRACIAS!" (CA 9).

"Muy enriquecedora. Importante establecer en el país estas instancias, que esperemos se continúen y diversifiquen. La posibilidad de participar de este curso abre la perspectiva de crecer y desarrollarse profesionalmente" (CA 14).

No se registraron quejas ni comentarios negativos acerca de la necesidad de viajar a Montevideo para participar de las instancias presenciales obligatorias. Es muy interesante destacar, sin embargo, que más de la mitad de los participantes (55\%) expresan que habrían optado por realizar el curso en modalidad presencial de haber podido.

Por último, el cambio en la modalidad de estudio significó, tanto para alumnos como para docentes y tutores, un cambio de paradigma, una experiencia nueva y removedora. Es natural que una experiencia así provoque la reflexión y el debate en el marco de la innovación y el cambio educativo.

\section{e. Lo afectivo}

El apoyo brindado por los docentes fue reconocido y valorado unánimemente. Este apoyo se canalizó por diversas vías, no solamente a través de Internet. Las siguientes citas de alumnos ilustran este aspecto:

"Me sentí muy bien. No sabía cómo resultaría, superó mis expectativas. [...] ¿Apoyo docente? No estaba acostumbrada a trabajar con ¡TANTO APOYO!" (CA 17).

Me sentí apoyada muy de cerca por los docentes, salvo en algún caso en particular" (CA 21).

En varios participantes aparece un sentimiento de aislamiento, en general al inicio del curso, aunque la mayoría expresó su sensación de pertenecer a un grupo y se declaró satisfecha por eso. La triangulación permite ver que existe una relación inversa entre la fluidez informática del participante y su percepción de aislamiento. En general, a menor fluidez informática, mayor aislamiento, lo cual es natural por tratarse de un curso a distancia a través de Internet. Un elemento que redujo la percepción de aislamiento fue el participar desde una localidad donde residían otros alumnos del postgrado.

A medida que cada participante se fue familiarizando con la tecnología, y gracias al apoyo de docentes y compañeros (brindado tanto a distancia como durante las instancias presenciales), en casi todos los casos esta sensación de aislamiento fue superada.

"Me sentí cómoda. La comunicación fue muy fluida y adecuada. Por tal motivo no me sentí aislada. El apoyo de los docentes fue permanente a pesar de la distancia física. Con respecto al grupo considero que el mismo siente identidad como tal. Todos nos sentimos integrantes del grupo de alumnos del curso a distancia. Nos reconocemos como pertenecientes al mismo. Y nos apoyamos a distancia cuando es necesario" (CA 6). 
Con respecto a la cohesión del grupo, se percibe como importante el haber contado con instancias presenciales. Éstas ofrecieron un aporte que trascendió la mera formación académica. Fueron fundamentales en el relacionamiento entre pares y docentes:

"En cuanto a los compañeros, las instancias presenciales fueron importantes para afianzar vínculos, más allá de esto al trabajar con otra compañera ya me hace sentir apoyada" (CA 1).

"Los seminarios presenciales fueron muy interesantes y precisos para dar un acercamiento a compañeros, docentes y a la institución" (CA 9).

En suma, el componente afectivo brindado por los docentes, tutores y personal administrativo, no solamente fue de recibo entre los participantes, sino que constituyó uno de los pilares sobre los que se basó la propuesta.

\section{Conclusiones y recomendaciones}

La edición estudiada del Diploma en Educación no solamente fue una experiencia valiosa en términos de desarrollo profesional y acceso a un postgrado de alta calidad para docentes de todo el país, sino una rica oportunidad de aprendizaje para el equipo de trabajo del Instituto de Educación y para la Universidad ORT.

Una de las particularidades de este postgrado radica en que sus alumnos, profesores y tutores tienen algo en común: todos son docentes. La tarea del docente tradicional ha sido descripta como "solitaria": se cierra la puerta del aula y el docente, solo, trabaja con sus estudiantes (Grünberg, 2001). El Diploma en Educación en modalidad a distancia puso de manifiesto, nuevamente, esa soledad, en este caso, en ambos extremos de la línea. Mostró la necesidad que unos y otros tienen de socializar, compartir, sentir la presencia del colega, docente o alumno a través de una discusión en el foro, de un mensaje de correo electrónico, en un chat, telefónicamente o en encuentros presenciales. El vínculo que un docente logra establecer con sus alumnos en esta modalidad puede volverse más fuerte que en el entorno presencial, a pesar de que la asociación rostro-nombre no siempre sea inmediata.

El cumplimiento de todos los requisitos de ingreso al postgrado es crítico para su correcto desarrollo. Esta experiencia mostró con claridad la necesidad de jerarquizar el entrenamiento en el uso de herramientas informáticas, tanto para los docentes como para los alumnos del postgrado. Esto permitiría incrementar las posibilidades de acceso a quienes no reúnen este requisito, necesidad que puede satisfacerse mediante la implementación de talleres presenciales, semi-presenciales o a distancia. Las ediciones posteriores del Diploma han incorporado este aspecto, así como muchas de las otras recomendaciones que surgen de este estudio.

Además de los requisitos de carácter tecnológico mencionados para los potenciales alumnos, los profesores a cargo de los seminarios y tutorías deben contar con un entrenamiento específico en el uso eficiente del correo electrónico, moderación de foros electrónicos y uso pedagógico del chat. La elección de los docentes para dictar un seminario a distancia cuya plataforma principal es Internet, implica, además de solvencia académica y un adecuado manejo de la tecnología, un compromiso con una modalidad diferente que supone tener el tiempo y el deseo de explorar e innovar.

El rol del docente en este escenario puede describirse como más directivo que en el aula presencial, con una tendencia a pautar el trabajo y las "entregas" de forma más estricta. También supone la revisión del discurso empleado: el texto de un mensaje enviado por correo electrónico o colocado en un foro puede ser sumamente frío y distante. Los docentes deben tener en cuenta el factor afectivo en su discurso. Esto mitiga el sentimiento de aislamiento que algunos estudiantes experimentan en esta modalidad. 
El foro de discusión puede ser interpretado como "una asamblea virtual asincrónica". Es la herramienta central del curso que suple los intercambios que se dan en una clase presencial. Un buen curso a distancia ofrecido a través de Internet supone un buen foro de discusión. Adicionalmente, el foro contribuye a mantener la cohesión del grupo. El acercamiento entre alumnos y docentes puede apuntalarse mediante la inclusión de fotografías, archivos de audio y video. Con el objetivo de favorecer la socialización y enriquecer las discusiones, puede pensarse en integrar a los alumnos de la edición presencial del Diploma en determinadas instancias de intercambio asincrónico que utilizan sus pares a distancia.

La necesidad un número importante de conexiones, aunque sean breves, surge con gran claridad, tanto para docentes como para participantes. Se valora la periodicidad de aportes a los foros por encima del largo de cada mensaje. La retroalimentación (feedback) sobre trabajos, aportes y consultas, debe de ser rápida.

Los chats fueron evaluadas muy positivamente por los participantes. La realización de más de un chat en cada seminario constituiría una mejora significativa. Sin perjuicio de esto, es recomendable que docentes y tutores ofrezcan oportunidades de encuentros presenciales, independientemente de los pautados en el cronograma del postgrado.

Las guías de aprendizaje y materiales de apoyo fueron muy bien evaluados por los participantes. Sin embargo, es posible mejorar significativamente su diseño y diagramación. La mejora puede centrarse en el uso de "lotes de texto manejables" y tareas cortas, tanto de corte asociativo como de corte reflexivo, focalizadas especialmente en la generación de conocimientos y no en su evaluación (West y Walsh, 1995). La inclusión de material dinámico con variados tipos de tareas de autoevaluación, así como la actualización regular de vínculos a sitios relevantes apuntalarían esta estrategia. La incorporación de tareas que inviten al participante a volcar reflexiones y aportes en el foro de debate de un modo sistemático (Salmon, 2001) fortalecería el "diálogo" entre el material estático (guías) y las comunicaciones, propiciando el ciclo lectura-reflexión-elaboración-discusión-lectura de un modo sencillo e integrado. Las ediciones posteriores del Diploma han incorporado estas recomendaciones con muy buenos resultados.

Los requisitos para la obtención del título "Diploma en Educación" para los egresados de las modalidades a distancia y presencial son los mismos. Los criterios y mecanismos de evaluación, por tanto, coinciden. Los trabajos de los alumnos a distancia de la edición estudiada mostraron la misma calidad académica que aquellos de sus pares presenciales.

Monografías, ediciones anteriores de los Cuadernos de Investigación Educativa, ponencias en coloquios y conferencias, entre otros materiales, constituyen recursos relevantes y útiles en este postgrado. Este material podría estar accesible a los potenciales participantes, pudiéndose utilizar, de este modo, no solamente como material de referencia, sino como herramienta de difusión del Diploma.

A lo largo de este estudio todos los actores, particularmente los docentes y tutores del Instituto de Educación, se mostraron rigurosamente autocríticos, sin perjuicio de las consecuencias que esta postura pudiera implicar. Primó la voluntad de realizar aportes que puedan traducirse en una mejora real de la propuesta educativa, sin considerar que en ese proceso, existía el riesgo de poner de manifiesto falencias propias.

Finalmente, debemos resaltar la función desarrollada por el Secretario Docente del Instituto de Educación, el Dr. Enrique Lorenzo, durante la planificación, desarrollo, ejecución y evaluación de este postgrado. Además de líder académico, docente y tutor, fue asesor clave en el marco de este estudio. La tarea por él desarrollada puede ser resumida en términos de "acompañamiento irrestricto" de alumnos, docentes, tutores e investigadores, pasando por los aspectos académicos, tecnológicos y de gestión. Vaya entonces nuestro homenaje a la inolvidable figura de Enrique. 


\section{Bibliografía}

Grünberg, J. 2000. Teachers and computer-mediated communication: a study of the development of collegiality among secondary school teachers using electronic mail. Doctoral dissertation, Keble College, Oxford University.

Salmon, G. 2000. E-Moderating: The key to teaching and learning online. Oxford: OUP.

West, R. \& Walsh, G. 1995. Inputs and Outputs of Distance Education, in Howard and McGrath (eds.) Distance education for language teachers. Clevedon: Multilingual Matters.

*Ph.D. en Tecnología Educativa, Universidad de Kent, Inglaterra. Master en Tecnología Educativa, Universidad de Manchester, Inglaterra. Learning Technologist, Unit for the Enhancement of Learning and Teaching, University of Kent at Canterbury. 